Pure and Applied Mathematics Quarterly

Volume 4, Number 1

(Special Issue: In honor of

Gregory Margulis, Part 2 of 2$)$

$81-97,2008$

\title{
Diophantine Properties of Measures and Homogeneous Dynamics
}

\author{
Dmitry Kleinbock
}

To G.A. Margulis on the occasion of his 60th birthday

\begin{abstract}
This is a survey of the so-called "quantitative nondivergence" approach to metric Diophantine approximation developed approximately 10 years ago in my collaboration with Margulis. The goal of this paper is to place the theory of approximation on manifolds into a broader context of studying Diophantine properties of points generic with respect to certain measures on $\mathbb{R}^{n}$. The correspondence between multidimensional Diophantine approximation and dynamics of lattices in Euclidean spaces is discussed in an elementary way, and several recent results obtained by means of this correspondence are surveyed.
\end{abstract}

\section{INTRODUCTION}

Let us start with the following definition. For $v>0$ and $n \in \mathbb{N}$, say that $\mathbf{y}=\left(y_{1}, \ldots, y_{n}\right) \in \mathbb{R}^{n}$ is $v$-approximable (notation: $\left.\mathbf{y} \in \mathcal{W}_{v}\right)$ if there are infinitely many $\mathbf{q}=\left(q_{1}, \ldots, q_{n}\right) \in \mathbb{Z}^{n}$ such that

$$
\left|y_{1} q_{1}+\cdots+y_{n} q_{n}+p\right|<\|\mathbf{q}\|^{-v}
$$

for some $p \in \mathbb{Z}$. Here we interpret $\mathbf{y} \in \mathbb{R}^{n}$ as row vectors, or linear forms. It will be convenient to represent integers $\mathbf{q} \in \mathbb{Z}^{n}$ as column vectors, so that $y_{1} q_{1}+\cdots+y_{n} q_{n}$ can be written as yq, and (1.1) as

$$
|\mathbf{y q}+p|<\|\mathbf{q}\|^{-v} \text {. }
$$

Hopefully such notation will cause no confusion. We remark that similarly one can consider the column vector version, the so-called "simultaneous approximation";

Received March 31, 2006.

Supported in part by NSF Grant DMS-0239463. 
most of the results mentioned in this paper have their simultaneous approximation analogues.

Then let us define the Diophantine exponent $\omega(\mathbf{y})$ of $\mathbf{y}$ by

$$
\omega(\mathbf{y}) \stackrel{\text { def }}{=} \sup \{v \mid \mathbf{y i s} v \text {-approximable }\} \text {. }
$$

Note that the above definition, unlike the previous one, does not depend on the choice of the norm $\|\cdot\|$. We will however always work with the sup-norm, $\|\mathbf{x}\|=\max _{i}\left|x_{i}\right|$.

The fact that $\omega(\mathbf{y})$ is always at least $n$ immediately follows from Dirichlet's Theorem, stating that the system

$$
\left\{\begin{array}{l}
|\mathbf{y q}+p|<1 / T \\
0<\|\mathbf{q}\| \leq T^{-1 / n}
\end{array}\right.
$$

has an integer solution $(p, \mathbf{q})$ for every $\mathbf{y} \in \mathbb{R}^{n}$ and $T>1$. Also it is not hard to see that $\omega(\mathbf{y})=n$ for $\lambda$-almost every $\mathbf{y} \in \mathbb{R}^{n}$, where $\lambda$ stands for Lebesgue measure on $\mathbb{R}^{n}$. Vectors $\mathbf{y}$ with $\omega(\mathbf{y})>n$ are usually called very well approximable (VWA).

Let us now extend the notion of Diophantine exponents to measures. Namely, if $\mu$ is a Radon measure on $\mathbb{R}^{n}$, let us define the Diophantine exponent $\omega(\mu)$ of $\mu$ to be the $\mu$-essential supremum of the function $\mathbf{y} \mapsto \omega(\mathbf{y})$. In other words,

$$
\omega(\mu) \stackrel{\text { def }}{=} \sup \{v \mid \mu(\{\mathbf{y} \mid \omega(\mathbf{y})>v\})>0\}=\sup \left\{v \mid \mu\left(\mathcal{W}_{v}\right)>0\right\} .
$$

Clearly it only depends on the measure class of $\mu$.

Very often (equivalence classes of) measures $\mu$ that we are going to consider will be naturally associated with subsets $\mathcal{M}$ of $\mathbb{R}^{n}$ supporting $\mu$. For example, if $\mathcal{M}$ is a smooth submanifold of $\mathbb{R}^{n}$, we will be taking (the class of) $\mu$ to be (that of) the Riemannian volume on $\mathcal{M}$, that is, the pushforward $\mathbf{f}_{*} \lambda$ of $\lambda$ by any smooth map $\mathbf{f}$ parametrizing $\mathcal{M}$. In this case we will define the Diophantine exponent $\omega(\mathcal{M})$ of $\mathcal{M}$ to be equal to that of $\mu$. From what was said it follows that $\omega(\mu) \geq n$ for any $\mu$, and $\omega(\lambda)=\omega\left(\mathbb{R}^{n}\right)$ is equal to $n$.

This justifies the terminology which has been introduced by V. Sprindžuk: let us say that a measure $\mu$ on $\mathbb{R}^{n}$ (resp. a subset $\mathcal{M}$ of $\mathbb{R}^{n}$ ) is extremal if $\omega(\mu)$ (resp. $\omega(\mathcal{M}))$ is equal to $n$, that is, attains the smallest possible value; equivalently, if $\mu$-a.e. $\mathbf{y} \in \mathbb{R}^{n}$ is not VWA.

The theory of Diophantine approximation on manifolds started with considering the map

$$
\mathbf{f}(x)=\left(x, x^{2}, \ldots, x^{n}\right) .
$$

The extremality of $\mathbf{f}_{*} \lambda$ for $\mathbf{f}$ as above was conjectured in 1932 by K. Mahler [M] and proved in 1964 by Sprindžuk [Sp1, Sp2]. At about the same time W. Schmidt 
[Sc1] proved the extremality of $\mathbf{f}_{*} \lambda$ when $\mathbf{f}: I \rightarrow \mathbb{R}^{2}, I \subset \mathbb{R}$, is $C^{3}$ and satisfies

$$
\left|\begin{array}{ll}
f_{1}^{\prime}(x) & f_{2}^{\prime}(x) \\
f_{1}^{\prime \prime}(x) & f_{2}^{\prime \prime}(x)
\end{array}\right| \neq 0 \quad \text { for } \lambda \text {-a.e. } x \in I \text {. }
$$

Since then, a lot of attention has been devoted to showing that measures $\mathbf{f}_{*} \lambda$ are extremal for other smooth maps $\mathbf{f}$.

To describe a broad class of examples, let us recall the following definition. Let $\mathbf{x} \in \mathbb{R}^{d}$ and let $\mathbf{f}=\left(f_{1}, \ldots, f_{n}\right)$ be a $C^{k}$ map from a neighborhood of $\mathbf{x}$ to $\mathbb{R}^{n}$. For $\ell \leq k$, say that $\mathbf{f}$ is $\ell$-nondegenerate at $\mathbf{x}$ if

$\mathbb{R}^{n}$ is spanned by partial derivatives of $\mathbf{f}$ at $\mathbf{x}$ of order up to $\ell$.

We will say that $\mathbf{f}$ is nondegenerate at $\mathbf{x}$ if (1.6) holds for some $\ell$. If $\mathcal{M}$ is a $d$-dimensional submanifold of $\mathbb{R}^{n}$, we will say that $\mathcal{M}$ is nondegenerate at $\mathbf{y} \in \mathcal{M}$ if any (equivalently, some) diffeomorphism $\mathbf{f}$ between an open subset $U$ of $\mathbb{R}^{d}$ and a neighborhood of $\mathbf{y}$ in $\mathcal{M}$ is nondegenerate at $\mathbf{f}^{-1}(\mathbf{y})$.

It was conjectured by Sprindžuk [Sp3] in 1980 that real analytic nondegenerate submanifolds of $\mathbb{R}^{n}$ are extremal. A number of special cases of this conjecture were established in subsequent years, but the general case stood open until the mid-1990s.

In Spring 1996, the following two events happened at Yale University. One came out of our work with Margulis [KM1, KM3] on bounded orbits and excursions to infinity of partially hyperbolic homogeneous flows. A substantial part of that activity had been motivated by problems in Diophantine approximation, and we were discussing possible number-theoretical applications of new results, in particular some generalizations of Dani's correspondence [D] between bounded orbits and badly approximable systems. The credit for the other event goes to Paul Lukasiewicz, the librarian of Yale Mathematics Department. As a result of his thoughtful arrangement of books in the AMS Translations Series on the library shelves, Sprindžuk's monograph [Sp2] happened to offer an unobstructed full frontal view of its title. The latter caught the eye of Margulis while he was passing by, prompting him to turn his attention to this circle of problems.

Shortly thereafter, a strengthened (with analytic replaced by $C^{k}$ for some $k$ ) version of Sprindžuk's conjecture was proved:

Theorem 1.1. [KM2] Let $\mathcal{M}$ be a d-dimensional smooth submanifold of $\mathbb{R}^{n}$ which is nondegenerate at its almost every point. Then $\mathcal{M}$ is extremal. Or, slightly more generally, if $U$ is an open subset of $\mathbb{R}^{d}$ and $\mathbf{f}: U \rightarrow \mathbb{R}^{n}$ is nondegenerate at $\lambda$-almost every point of $U$, then $\mathbf{f}_{*} \lambda$ is extremal. 
The method of proof in [KM2] was dynamical in nature ${ }^{1}$, and the main purpose of this note is to explain its main ideas and a possibility to use them to solve more general problems. Speaking of which, it seems natural not to restrict oneself to smooth measures on submanifolds, and thus ask

Question 1.2. What other measures on $\mathbb{R}^{n}$ can be shown to be extremal?

In fact, pushing it even further, one can ask

Question 1.3. For what other measures $\mu$ on $\mathbb{R}^{n}$ can one compute or estimate $\omega(\mu)$ ?

The present paper, among other things, provides answers to both questions. The main tool is a dynamical description of Diophantine exponents of vectors/measures described in $\S 2$. In $\S 3$ it is shown how that description can turn the quantitative nondivergence phenomenon, dating back to the work of Margulis in early 1970s, into a proof of Theorem 1.1. A partial list of further applications of the method and related open questions is given in the last section of the paper.

Acknowledgements. Needless to say, I am immensely grateful to Gregory Margulis for all his encouragement and guidance before, during and after our collaboration, and am very happy to join other contributors to this volume to wish him Happy Birthday and many more theorems, papers and graduate students in years to come.

The exposition of the present paper roughly follows the author's talk given at the conference on "Diophantine analysis, uniform distributions and applications" in Minsk, Belarus in August 2003; a preliminary shorter version of this note has been submitted to the conference proceedings. The hospitality of the organizers of this conference is gratefully acknowledged. Thanks are also due to Victor Beresnevich and Barak Weiss for useful comments.

\section{Diophantine APproximation AND DYNAmics}

The correspondence between Diophantine properties of vectors in $\mathbb{R}^{n}$ and dynamical properties of lattices in $\mathbb{R}^{n+1}$ dates back for $n=1$ to E. Artin, and for $n>1$ to the work of Davenport-Schmidt [DS2], Schmidt [Sc2] and Dani [D]. Here we present a condensed exposition of the main principle behind a reduction of Theorem 1.1 to a dynamical statement. Note that a similar exposition can be found in survey papers [K1] and [Ma2], as well as in Chapter IV of [St].

Let us pick $v_{0} \geq n$ and $\mathbf{y} \in \mathbb{R}^{n}$ with $\omega(\mathbf{y})>v_{0}$. According to the definition, this means that for some $v>v_{0}$ inequality (1.2) is satisfied for infinitely many

\footnotetext{
${ }^{1}$ Later an alternative proof was found by Beresnevich [Be].
} 
$(p, \mathbf{q})$. Equivalently, for infinitely many $s \in \mathbb{N}$ the following system has an integer solution $(p, \mathbf{q})$ :

$$
\left\{\begin{array}{l}
|\mathbf{y q}+p|<2^{-v s} \\
2^{s} \leq\|\mathbf{q}\|<2^{s+1}
\end{array} .\right.
$$

We will drop the first of the inequalities in the second line, and conclude that $\omega(\mathbf{y})>v_{0}$ implies that for some $v>v_{0}$ there exist infinitely many $s \in \mathbb{N}$ such that the system

$$
\left\{\begin{array}{l}
|\mathbf{y q}+p|<2^{-v s} \\
\|\mathbf{q}\|<2^{s+1}
\end{array}\right.
$$

has an integer solution $(p, \mathbf{q})$ with $\mathbf{q} \neq 0$.

The latter system can be conveniently written in a matrix form. Namely, y gives rise to

$$
u_{\mathbf{y}} \stackrel{\text { def }}{=}\left(\begin{array}{ll}
1 & \mathbf{y} \\
0 & I_{n}
\end{array}\right),
$$

and the right hand sides of the inequalities in (2.1) define a certain rectangular box in $\mathbb{R}^{n+1}$, namely

$$
B_{v, s}=\left\{\left(x_{0}, x_{1}, \ldots, x_{n}\right)|| x_{0}\left|<2^{-v s},\right| x_{i} \mid<2^{s+1} \text { for } i=1, \ldots, n\right\} .
$$

Thus $\omega(\mathbf{y})>v_{0}$ implies that for some $v>v_{0}$ there exist infinitely many $s \in \mathbb{N}$ such that $u_{\mathbf{y}} \mathbb{Z}^{n+1} \cap B_{v, s} \neq\{0\}$.

The next step is to transform this box into a cube, which naturally would be easier to work with than $B_{v, s}$. This is where dynamics comes into play. There is only one way to undertake such a transformation, preserving the volume: one needs to act by

$$
g_{t}=\operatorname{diag}\left(2^{n t}, 2^{-t}, \ldots, 2^{-t}\right) .
$$

Note that the volume of $B_{v, s}$ is equal to $2^{2 n+1-(v-n) s}$, which tends to 0 when $s \rightarrow \infty$ (recall that $v$ is chosen to be bigger than $v_{0} \geq n$ ). If for any $s$ one chooses $t>0$ such that $g_{t} B_{v, s}$ is a cube, then it is clear that the sidelength of this cube will be very small for large $s$. In fact, an elementary computation shows that $s$ and $t$ in this situation are not far from each other, and the sidelength of $g_{t} B_{v, s}$ is equal to $C_{n} 2^{-\gamma t}$, where $C_{n}$ is an explicit constant depending only on $n$ and

$$
\gamma=\gamma(v)=\frac{v-n}{n(v+1)} .
$$

We have almost proved 
Proposition 2.1. Suppose that $\omega(\mathbf{y})>v_{0}$ for some $\mathbf{y} \in \mathbb{R}^{n}$ and $v_{0} \geq n$. Then for some $\gamma>\gamma\left(v_{0}\right)$ there exist infinitely many $t \in \mathbb{N}$ such that

$$
\text { the lattice } g_{t} u_{\mathbf{y}} \mathbb{Z}^{n+1} \text { has a nonzero vector of norm less than } 2^{-\gamma t} \text {. }
$$

Proof. It only remains to observe that taking $\gamma$ between $\gamma(v)$ and $\gamma\left(v_{0}\right)$ allows one to get rid of the constant $C_{n}$, as well as to replace every $t$ chosen as above by its integer part.

In fact, the converse to this proposition is also true and easy to prove, see [K2] or [KM3], but it will not be needed here.

It might be helpful for the understanding to discuss the geometric meaning of the conclusion of the above proposition. Denote by $\Omega_{k}$ the space of lattices in $\mathbb{R}^{k}$ of covolume 1, and let

$$
\Omega_{k}(\varepsilon) \stackrel{\text { def }}{=}\left\{\Lambda \in \Omega_{k} \mid\|\mathbf{v}\|<\varepsilon \text { for some } \mathbf{v} \in \Lambda \backslash\{0\}\right\} .
$$

Then (2.3) can be written as $g_{t} u_{\mathbf{y}} \mathbb{Z}^{n+1} \in \Omega_{n+1}\left(2^{-\gamma t}\right)$. It is well known that $\Omega_{k}$ is noncompact, but the complement of $\Omega_{k}(\varepsilon)$ in $\Omega_{k}$ is compact for any positive $\varepsilon$, and, further, any bounded subset of $\Omega_{k}$ belongs to such a complement for some $\varepsilon>0$ (Mahler's Compactness Criterion, see $[R]$ or $[\mathrm{BM}]$ ). Thus vectors with large Diophantine exponents give rise to $g_{t}$-trajectories in the space of lattices with "fast enough growth". See [KM2, K1, K2] for more details.

Here is an application of Proposition 2.1:

Corollary 2.2. Let $U$ be an open subset of $\mathbb{R}^{d}, \mu$ a measure on $U$, and let $\mathbf{f}$ be a map from $U$ to $\mathbb{R}^{n}$. Take $v \geq n$, and suppose that for $\mu$-a.e. $\mathbf{x}_{0} \in U$ one can find a ball $B \subset U$ centered in $\mathbf{x}_{0}$ such that for any $\gamma>\gamma(v)$ one has

$$
\sum_{t=1}^{\infty} \mu\left(\left\{\mathbf{x} \in B \mid g_{t} u_{\mathbf{f}(\mathbf{x})} \mathbb{Z}^{n+1} \in \Omega_{n+1}\left(2^{-\gamma t}\right)\right\}\right)<\infty .
$$

Then $\omega\left(\mathbf{f}_{*} \mu\right) \leq v$.

Proof. Indeed, in view of the Borel-Cantelli Lemma, (2.4) implies that

$$
\mu\left(\left\{\mathbf{x} \in B \mid g_{t} u_{\mathbf{f}(\mathbf{x})} \mathbb{Z}^{n+1} \in \Omega_{n+1}\left(2^{-\gamma t}\right) \text { for infinitely many } t\right\}\right)=0 .
$$

Hence it follows from the assumption and Proposition 2.1 that for $\mu$-a.e. $\mathbf{x}_{0} \in U$ one can find a ball $B$ centered in $\mathbf{x}_{0}$ such that $\left.\mu(\mathbf{x} \in B \mid \omega(\mathbf{f}(\mathbf{x}))>v\}\right)=0$, and the latter, in view of the definition (1.4), implies that $\omega(\mu) \leq v$.

Summarizing the above discussion, we can observe that an upper estimate for $\omega\left(\mathbf{f}_{*} \mu\right)$, and in particular the extremality of $\mathbf{f}_{*} \mu$, can be derived from knowing that sets of the form

$$
\left\{\mathbf{x} \in B \mid g_{t} u_{\mathbf{f}(\mathbf{x})} \mathbb{Z}^{n+1} \in \Omega_{n+1}(\varepsilon)\right\}
$$


have small enough measure. In other words, the $g_{t}$-translate of the pushforward of $\mu$ by the map $\mathbf{x} \mapsto u_{\mathbf{f}(\mathbf{x})} \mathbb{Z}^{n+1}, B \rightarrow \Omega_{n+1}$, does not assign too much weight to the "neighborhood of infinity" $\Omega_{n+1}(\varepsilon)$ in the space of lattices. The latter is precisely a consequence of so-called quantitative nondivergence estimates, to be discussed in the next section.

\section{Quantitative Nondivergence AND PRoOF OF Theorem 1.1}

In order to state a general result which can be used to estimate the measure of sets (2.5), we first introduce some notation and definitions. For a ball $B=B(\mathbf{x}, r)$ in $\mathbb{R}^{n}$ and $a>0$, we denote $B(\mathbf{x}, a r)$ by $a B$. If $B$ is a subset of $\mathbb{R}^{n}$ and $f$ is a real-valued function on $\mathbb{R}^{n}$, we let

$$
\|f\|_{B} \stackrel{\text { def }}{=} \sup _{\mathbf{x} \in B}|f(\mathbf{x})|
$$

and if $\mu$ is a measure on $\mathbb{R}^{n}$ such that $\mu(B)>0$, we define $\|f\|_{\mu, B}$ to be equal to $\|f\|_{B \cap \operatorname{supp} \mu}$.

Given $D \geq 1$, say that a measure $\mu$ on $\mathbb{R}^{n}$ is $D$-Federer on an open $U \subset \mathbb{R}^{n}$ if

$$
\mu(3 B) \leq D \mu(B)
$$

for every ball $B$ centered in supp $\mu$ with $3 B \subset U$. We will say that $\mu$ is $D$-Federer if for $\mu$-a.e. point of $\mathbb{R}^{n}$ there exists a neighborhood $U$ of this point such that $\mu$ is $D$-Federer on $U$. In fact, for most of subsequent applications a non-uniform version will suffice: $\mu$ will be called Federer if for $\mu$-a.e. $\mathbf{x} \in \mathbb{R}^{n}$ there exist a neighborhood $U$ of $\mathbf{x}$ and $D>0$ such that $\mu$ is $D$-Federer on $U$.

Clearly $\lambda$ and, more generally, volume measures on smooth submanifolds satisfy the above conditions. But many other natural measures can also be proved to be Federer. See [KLM, KW1, MU, S] for examples.

The next definition involves an important property of certain functions $f$ with respect to certain measures $\mu$. Given $C, \alpha>0$, a subset $U$ of $\mathbb{R}^{n}$, a measure $\mu$ on $U$ and a real-valued function $f$ on $U$, say that $f$ is $(C, \alpha)$-good on $U$ with respect to $\mu$ if for any open ball $B \subset U$ centered in supp $\mu$ and any $\varepsilon>0$ one has

$$
\mu(\{x \in B|| f(x) \mid<\varepsilon\}) \leq C\left(\frac{\varepsilon}{\|f\|_{\mu, B}}\right)^{\alpha} \mu(B) .
$$

The primary example is given by polynomial maps. See [KM2, BKM, KLM], as well as $\S 4.1$ of the present paper, for various other examples.

We are now ready to state our main estimate. It was proved in [KM2] (Theorem $5.4)$ in the case $\mu=\lambda$, and then generalized in $[\mathrm{KLM}]$ and $[\mathrm{KT}]$. 
Theorem 3.1. For $d, n \in \mathbb{N}$, let a ball $B \subset \mathbb{R}^{d}$, a measure $\mu$ on $\mathbb{R}^{d}$ such that $B$ is centered in supp $\mu$ and $\mu$ is D-Federer on $\tilde{B} \stackrel{\text { def }}{=} 3^{n+1} B$, and a continuous map $\mathbf{f}=\left(f_{1}, \ldots, f_{n}\right): \tilde{B} \rightarrow \mathbb{R}^{n}$ be given. Suppose also that for some $C, \alpha>0$ and $0<\varrho<1$ the following two conditions hold:

(i) for any $\mathbf{c}=\left(c_{0}, c_{1}, \ldots, c_{n}\right) \in \mathbb{R}^{n+1}$, the function $c_{0}+\sum_{i=1}^{n} c_{i} f_{i}$ is $(C, \alpha)$ good on $\tilde{B}$ w.r.t. $\mu$;

(ii) for any $\mathbf{c} \in \mathbb{R}^{n+1}$ with $\|\mathbf{c}\| \geq 1,\left\|c_{0}+\sum_{i=1}^{n} c_{i} f_{i}\right\|_{\mu, B} \geq \varrho$.

Then for any positive $\varepsilon \leq \varrho$ and any $t>0$ one has

$$
\mu\left(\left\{\mathbf{y} \in B \mid g_{t} u_{\mathbf{y}} \mathbb{Z}^{n+1} \in \Omega_{n+1}(\varepsilon)\right\}\right) \leq E\left(\frac{\varepsilon}{\varrho}\right)^{\alpha} \mu(B),
$$

where $E$ is an explicit constant depending on $d, n, C, \alpha$ and $D$.

The proof of this theorem is not easy. But fortunately most of it has been around since the early 1970s, when Margulis [Ma1] proved that unipotent flows on the space of lattices do not go to infinity. In fact, his proof applies verbatim if one replaces a unipotent subgroup by a polynomial map. Later it was realized that the only way the polynomiality of the map is used is via the $(C, \alpha)$-good property, and that it can also produce a quantitative strengthening of non-divergence to infinity, namely an estimate for a relative measure of the intersection of the trajectory with a small "neighborhood of infinity" in the space of lattices.

Let us now show how this theorem can be applied to Diophantine approximation. Let a measure $\mu$ on $\mathbb{R}^{d}, \mathbf{x} \in \operatorname{supp} \mu$, and a map $\mathbf{f}=\left(f_{1}, \ldots, f_{n}\right)$ from a neighborhood of $\mathbf{x}$ to $\mathbb{R}^{n}$ be given.

The following definitions will be helpful. Let us say that the pair $(\mathbf{f}, \mu)$ is

- $(C, \alpha)-\operatorname{good}$ at $\mathbf{x}$ if

there exists a neighborhood $V \subset U$ of $\mathbf{x}$ such that

any linear combination of $1, f_{1}, \ldots, f_{n}$ is $(C, \alpha)$-good on $V$ w.r.t. $\mu$;

- good at $\mathbf{x}$ if it is $(C, \alpha)$-good at $\mathbf{x}$ for some $C, \alpha$;

- nonplanar at $\mathbf{x}$ if

for any neighborhood $B$ of $\mathbf{x}$

the restrictions of $1, f_{1}, \ldots, f_{n}$ to $B \cap \operatorname{supp} \mu$

are linearly independent over $\mathbb{R}$;

in other words, if $\mathbf{f}(B \cap \operatorname{supp} \mu)$ is not contained in any proper affine subspace of $\mathbb{R}^{n}$.

We can now prove 
Theorem 3.2. Let $\mu$ be a Federer measure on $\mathbb{R}^{d}, U$ an open subset of $\mathbb{R}^{d}$, and $\mathbf{f}: U \rightarrow \mathbb{R}^{n}$ a continuous map such that $(\mathbf{f}, \mu)$ is good and nonplanar at $\mu$-almost every point of $U$. Then $\mathbf{f}_{*} \mu$ is extremal.

Proof. Take $\mathbf{x} \in U \cap \operatorname{supp} \mu$ and $C, \alpha>0$ such that (3.1) and (3.2) hold, and let $B$ be a ball centered at $\mathbf{x}$ such that $3^{n+1} B$ is contained in $V$. Then condition (i) of Theorem 3.1 will be satisfied for $C, \alpha$ as above, and the existence of $\varrho>0$ satisfying (ii) follows from the compactness of the unit sphere in $\mathbb{R}^{n+1}$. Thus Theorem 3.1 applies, and it follows that for any small enough positive $\varepsilon$ and any $t>0$ one has

$$
\mu\left(\left\{\mathbf{x} \in B \mid g_{t} u_{\mathbf{f}(\mathbf{x})} \mathbb{Z}^{n+1} \in \Omega_{n+1}(\varepsilon)\right\}\right) \leq \mathrm{const} \cdot \varepsilon^{\alpha},
$$

with the constant independent of $\varepsilon$ or $t$. Putting $\varepsilon=e^{-\gamma t}$ for an arbitrarily small $\gamma>0$ and using Corollary 2.2 finishes the proof.

The above theorem first appeared in $[\mathrm{KLM}]$ in a slightly disguised version: there $n$ was equal to $d, \mathbf{f}$ was the identity map, and conditions sufficient for extremality were stated in terms of $\mu$. But the proof given there (which itself is a generalization of the argument from [KM2]) in fact easily yields the result stated above. The argument was generalized even further in $[\mathrm{KT}]$, where the wording was similar to that of the present paper.

Note also that by definition, the set

$$
\{\mathbf{x} \mid(\mathbf{f}, \mu) \text { is nonplanar at } \mathbf{x}\}
$$

is closed, so the nonplanarity at $\mu$-almost every point is equivalent to the same at every $\mathbf{x} \in U \cap \operatorname{supp} \mu$.

In order to see that Theorem 1.1 is a special case of Theorem 3.2, it suffices to show that for a smooth map $\mathbf{f}: U \rightarrow \mathbb{R}^{n},(\mathbf{f}, \lambda)$ is good and nonplanar at every point where $\mathbf{f}$ is nondegenerate. The nonplanarity is straightforward (indeed, the nondegeneracy of $\mathbf{f}$ at $\mathbf{x}$ clearly implies the existence of a neighborhood $B \ni \mathbf{x}$ such that $\mathbf{f}(B)$ is not contained in any proper affine subspace of $\left.\mathbb{R}^{n}\right)$. And the $(C, \alpha)$-good property of linear combinations of $1, f_{1}, \ldots, f_{n}$ basically follows from the fact that locally $\mathbf{f}$ can be approximated by a polynomial map, and is proved in [KM2].

It seems to be worthwhile to compare the method of proof of Theorem 1.1 discussed above with the standard approach based on Sprindžuk's solution of Mahler's problem and carried out in [Be]. As we have seen, the correspondence between Diophantine approximation and dynamics is quite natural and easy to explain. Also, the crucial measure estimate (Theorem 3.1) is the only hard part of the argument, the rest is relatively easy. Another advantage is a chance to work with non-smooth objects - we will mention in the next section how Theorem 3.2 gives rise to a wide variety of examples of extremal measures which are not 
volume measures on smooth submanifolds. Further, as will also be discussed in the next section, the dynamical approach can be perturbed in many directions and allows many generalizations and modifications of the result proved above.

However, the standard methods have a number of obvious advantages as well. The dynamical approach is hard to use when more precise results are needed, for example when the goal is to prove the divergence case of Khintchine-type theorems, or compute/estimate the Hausdorff dimension of the set of $v$-approximable points on a manifold. See [Be, BDV1, BDV2, VV] for examples of such results. Roughly speaking, the correspondence between approximation and dynamics is powerful but coarse, so that a substantial amount of information is being lost in transmission.

\section{Beyond THEOREM 1.1}

4.1. As was mentioned above, one of the main advantages of the method is that the assumptions of Theorem 3.2 are much less restrictive that those of Theorem 1.1. Here is an example. Following [KLM], given $C, \alpha>0$ and $U \subset \mathbb{R}^{d}$, say that $\mu$ is absolutely $(C, \alpha)$-decaying on $U$ if for any non-empty open ball $B \subset U$ of radius $r$ centered in supp $\mu$, any affine hyperplane $\mathcal{L} \subset \mathbb{R}^{n}$, and any $\varepsilon>0$ one has

$$
\mu\left(B \cap \mathcal{L}^{(\varepsilon)}\right) \leq C\left(\frac{\varepsilon}{r}\right)^{\alpha} \mu(B),
$$

where $\mathcal{L}^{(\varepsilon)}$ stands for the $\varepsilon$-neighborhood of $\mathcal{L}$. We will say that a measure is absolutely decaying if for $\mu$-a.e point of $\mathbb{R}^{d}$ there exist a neighborhood $U$ of this point and $C, \alpha>0$ such that $\mu$ is absolutely $(C, \alpha)$-decaying on $U$. Measures which are absolutely decaying and Federer were called absolutely friendly in [PV], see $[\mathrm{KLM}]$ for justification of this terminology.

If $\mu$ is absolutely decaying, it easily follows that

$$
\mu(\mathcal{L})=0 \text { for any affine hyperplane } \mathcal{L} \subset \mathbb{R}^{n}
$$

(i.e., in the terminology introduced in $\S 3,\left(\operatorname{Id}: \mathbb{R}^{n} \rightarrow \mathbb{R}^{n}, \mu\right)$ is nonplanar at every point of supp $\mu$ ). It also follows that $(\mathrm{Id}, \mu)$ is good at $\mu$-almost every point. In $[\mathrm{KLM}]$, much more than that has been proved:

Proposition 4.1. Let $\mu$ be an absolutely friendly measure on $U \subset \mathbb{R}^{d}$, and let $\mathbf{f}: U \rightarrow \mathbb{R}^{n}$ be a $C^{\ell+1}$ map which is $\ell$-nondegenerate at $\mathbf{x}_{0} \in U$. Then $(\mathbf{f}, \mu)$ is (a) good and (b) nonplanar at $\mathbf{x}_{0}$.

Part (b) is straightforward from (4.1), but part (a) is nontrivial and can be thought of as a generalization of the case $\mu=\lambda$ worked out in [KM2]. However note that the method of proof is completely different and has an advantage of producing a better esponent $\alpha$ in many cases, although there is a slight disadvantage of requiring an extra derivative. 
The above proposition, combined with Theorem 3.2, immediately implies that $\mathbf{f}_{*} \mu$ is extremal whenever $\mu$ is absolutely friendly and $\mathbf{f}$ is nondegenerate. The result is interesting even when $\mathbf{f}$ is the identity map (see $[\mathrm{PV}]$ for an alternative proof of the latter special case). Indeed, one can exhibit a wide variety of absolutely friendly measures supported on very peculiar sets, such as self-similar or self-conformal fractals. The prime example is the middle-third Cantor set $\mathcal{C}$ on the real line: its extremality (or, more precisely, the extremality of the natural measure $\mu_{\mathcal{C}}$ it supports) was established by Barak Weiss in [W], and later in $[\mathrm{KLM}]$ higher dimensional generalizations of $\mu_{\mathcal{C}}$ were shown to satisfy conditions sufficient for extremality. More examples have been recently found by M. Urbański [U1, U2], see also [SU].

4.2. The dynamical approach is indispensable in handling the so-called multiplicative generalization of the problems discussed in the introduction. Namely, define $\Pi_{+}(\mathbf{q}) \stackrel{\text { def }}{=} \prod_{q_{i} \neq 0}\left|q_{i}\right|$, say that $\mathbf{y}=\left(y_{1}, \ldots, y_{n}\right) \in \mathbb{R}^{n}$ is $v$-multiplicatively approximable (notation: $\mathbf{y} \in \mathcal{W}_{v}^{\times}$) if there are infinitely many $\mathbf{q} \in \mathbb{Z}^{n}$ such that

$$
|\mathbf{y q}+p|<\Pi_{+}(\mathbf{q})^{-v / n}
$$

for some $p \in \mathbb{Z}$, and then define multiplicative Diophantine exponents:

- $\omega^{\times}(\mathbf{y})$ of $\mathbf{y}$ by $\omega^{\times}(\mathbf{y}) \stackrel{\text { def }}{=} \sup \left\{v \mid \mathbf{y} \in \mathcal{W}_{v}^{\times}\right\}$,

- $\omega^{\times}(\mu)$ of $\mu$ by $\omega^{\times}(\mu) \stackrel{\text { def }}{=} \sup \left\{v \mid \mu\left(\mathcal{W}_{v}^{\times}\right)>0\right\}$.

It is easy to see that $\omega^{\times}(\mathbf{y})$ is not less than $\omega(\mathbf{y})$ for all $\mathbf{y}$, and yet $\omega^{\times}(\mathbf{y})=n$ for $\lambda$-a.e. $\mathbf{y} \in \mathbb{R}^{n}$, that is, $\omega^{\times}(\lambda)=n$. Following Sprindžuk, say that $\mu$ is strongly extremal if $\omega^{\times}(\mu)=n$. The multiplicative analogue of Theorem 1.1 (more precisely, of its real analytic version) was conjectured by Sprindžuk in 1980 and proved in [KM2]. Likewise, the following can be proved:

Theorem 4.2. Let $\mu$ and $\mathbf{f}$ be as in Theorem 3.2. Then $\mathbf{f}_{*} \mu$ is strongly extremal.

Thus in all the examples mentioned in $\S 4.1, \mathbf{f}_{*} \mu$ actually happens to be strongly extremal. The proof of the stronger statement is based on using the multiparameter action of

$$
g_{\mathbf{t}}=\operatorname{diag}\left(2^{t}, 2^{-t_{1}}, \ldots, 2^{-t_{n}}\right), \quad \text { where } \quad t=t_{1}+\cdots+t_{n},
$$

instead of (2.2).

4.3. Obvious examples of non-extremal manifolds are provided by proper affine subspaces of $\mathbb{R}^{n}$ whose coefficients are well enough approximable by rational numbers. On the other hand, it is clear from a Fubini argument that almost all translates of any given subspace are extremal. In [K2] the method of [KM2] was pushed further to produce criteria for the extremality, as well as the strong 
extremality, of an affine subspace $\mathcal{L}$ of $\mathbb{R}^{n}$. Further, it was shown that if $\mathcal{L}$ is extremal (resp. strongly extremal), then so is any smooth submanifold of $\mathcal{L}$ which is nondegenerate in $\mathcal{L}$ at its a.e. point. (The latter property is a straightforward generalization of the definition of nondegeneracy in $\mathbb{R}^{n}$ : a map $\mathbf{f}$ is nondegenerate in $\mathcal{L}$ at $\mathbf{x}$ if the linear part of $\mathcal{L}$ is spanned by partial derivatives of $\mathbf{f}$ at $\mathbf{x}$.) In other words, extremality and strong extremality pass from affine subspaces to their nondegenerate submanifolds.

A more precise analysis makes it possible to study Diophantine exponents of measures with supports contained in arbitrary proper affine subspaces of $\mathbb{R}^{n}$. Namely, in [K4] it is shown how to compute $\omega(\mathcal{L})$ for any $\mathcal{L}$. Furthermore, the following generalization of Theorem 3.2 is obtained:

Theorem 4.3. Let $\mu$ be a Federer measure on $\mathbb{R}^{d}, U$ an open subset of $\mathbb{R}^{d}, \mathcal{L}$ an affine subspace of $\mathbb{R}^{n}$, and let $\mathbf{f}: U \rightarrow \mathcal{L}$ be a continuous map such that $(\mathbf{f}, \mu)$ is good and nonplanar in $\mathcal{L}$ at $\mu$-almost every point of $U$. Then $\omega\left(\mathbf{f}_{*} \mu\right)=\omega(\mathcal{L})$.

Here we say that $(\mathbf{f}, \mu)$ is nonplanar in $\mathcal{L}$ at $\mathbf{x}$ if for any neighborhood $B$ of $\mathbf{x}$, the $\mathbf{f}$-image of $B \cap \operatorname{supp} \mu$ is not contained in any proper affine subspace of $\mathcal{L}$, thus generalizing the definition from $\S 3$. It is easy to see that for a smooth map $\mathbf{f}: U \rightarrow \mathcal{L},(\mathbf{f}, \lambda)$ is good and nonplanar in $\mathcal{L}$ at every point at which $\mathbf{f}$ is nondegenerate in $\mathcal{L}$.

4.4. Another application concerns badly approximable vectors. Recall that $\mathbf{y} \in$ $\mathbb{R}^{n}$ is called badly approximable if there exists $c>0$ such that for any $\mathbf{q} \in \mathbb{Z}^{n} \backslash\{0\}$ and $p \in \mathbb{Z}$ one has

$$
|\mathbf{y q}+p|>c\|\mathbf{q}\|^{-n}
$$

Denote the set of badly approximable vectors in $\mathbb{R}^{n}$ by $\mathcal{B A}$. It is a theorem of Dani $[\mathrm{D}]$ that $\mathbf{y} \in \mathcal{B A}$ if and only if the trajectory

$$
\left\{g_{t} u_{\mathbf{y}} \mathbb{Z}^{n+1} \mid t>0\right\}
$$

where $g_{t}$ and $u_{\mathbf{y}}$ are as in $\S 2$, does not intersect $\Omega_{n+1}(\varepsilon)$ for some $\varepsilon>0$, i.e. is bounded in $\Omega_{n+1}$.

Using this and Theorem 3.1 it turns out to be possible to find badly approximable vectors inside supports of certain measures on $\mathbb{R}^{n}$. Here is one way to state the result of the paper [KW1]. Denote by $\operatorname{dim}(K)$ the Hausdorff dimension of a subset $K$ of $\mathbb{R}^{n}$, and for $\beta>0$ let us say that a measure $\mu$ on $\mathbb{R}^{n}$ is $\beta$-scaling if there is a positive $c>0$ such that for every ball $B$ of radius $r$ one has $\mu(B) \leq c r^{\beta}$. It is well known (Mass Distribution Principle + Frostman's Lemma) that

$$
\operatorname{dim}(K)=\sup \{\beta \mid K \text { supports a } \beta \text {-scaling measure }\} .
$$


Now let us define

$\operatorname{dim}_{a f}(K) \stackrel{\text { def }}{=} \sup \{\beta \mid K$ supports a $\beta$-scaling absolutely friendly measure $\}$.

Naturally, it is always not bigger than $\operatorname{dim}(K)$ (but can be much less). The following is shown in [KW1] (see also [KTV] for a similar result):

Theorem 4.4. For any compact subset $K$ of $\mathbb{R}^{n}$, one has

$$
\operatorname{dim}_{a f}(K \cap \mathcal{B A}) \geq \operatorname{dim}_{a f}(K) .
$$

In particular, if a set $K$ of Hausdorff dimension $\beta$ supports an absolutely friendly $\beta$-scaling measure (and many examples of such sets have been found in $[\mathrm{KLM}, \mathrm{KW} 1, \mathrm{U} 1, \mathrm{U} 2, \mathrm{SU}])$, then $\operatorname{dim}(K \cap \mathcal{B \mathcal { A }})=\beta$.

4.5. For positive $\varepsilon<1$, say that Dirichlet's Theorem can be $\varepsilon$-improved for $\mathbf{y} \in \mathbb{R}^{n}$, writing $\mathbf{y} \in \mathcal{D} \mathcal{I}_{\varepsilon}$, if for every sufficiently large $T$ the system

$$
\left\{\begin{array}{l}
|\mathbf{y q}+p|<\varepsilon / T \\
0<\|\mathbf{q}\|<\varepsilon T^{1 / n}
\end{array}\right.
$$

(that is, (1.3) with the right hand side terms multiplied by $\varepsilon$ ) has an integer solution $(p, \mathbf{q})$. This is easily seen to be equivalent to

$$
g_{t} u_{\mathbf{y}} \mathbb{Z}^{n+1} \in \Omega_{n+1}(\varepsilon) \text { for large enough } t .
$$

Since the complement to $\Omega_{n+1}(\varepsilon)$ has nonempty interior for any $\varepsilon<1$, it follows from the ergodicity of the $g_{t}$-action on $\Omega_{n+1}$ that

$$
\lambda\left(\mathcal{D} \mathcal{I}_{\varepsilon}\right)=0 \text { for any } \varepsilon<1 .
$$

The above is roughly the outline of the proof of (4.2) by Davenport and Schmidt [DS2], who gave a direct proof of density of $g_{t}$-orbits of almost all lattices, without reference to ergodicity.

Similar questions with $\lambda$ replaced by $\mathbf{f}_{*} \lambda$ for some specific smooth maps $\mathbf{f}$ were considered in [DS1, Ba1, Ba2, DRV, Bu]. For example, [Bu,Theorem 7]provides an explicitly computable constant $c=c(n)$ such that for $\mathbf{f}$ as in (1.5),

$$
\mathbf{f}_{*} \lambda\left(\mathcal{D I} \mathcal{I}_{\varepsilon}\right)=0 \text { for } \varepsilon<c .
$$

This had been previously done in [DS1] for $n=2$ and in [Ba1] for $n=3$.

In [KW3] we use Theorem 3.1 to generalize this to a much broader class of measures:

Theorem 4.5. Given $d, n \in \mathbb{N}$ and $C, \alpha, D>0$, there exists $c=c(d, n, C, \alpha, D)$ with the following property. Let $\mu$ be a D-Federer measure on $\mathbb{R}^{d}, U$ an open subset of $\mathbb{R}^{d}$, and $\mathbf{f}: U \rightarrow \mathbb{R}^{n}$ a continuous map such that $(\mathbf{f}, \mu)$ is $(C, \alpha)$-good and nonplanar at $\mu$-almost every point of $U$. Then $\mathbf{f}_{*} \mu\left(\mathcal{D} \mathcal{I}_{\varepsilon}\right)=0$ for $\varepsilon<c$. 
In particular, almost every point on any nondegenerate smooth manifold is not in $\mathcal{D} \mathcal{I}_{\varepsilon}$ for small enough $\varepsilon$. See also [KW2] for a similar result about the set of singular vectors, defined as the intersection of $\mathcal{D} \mathcal{I}_{\varepsilon}$ over all positive $\varepsilon$; those correspond to divergent $g_{t}$-trajectories.

4.6. In all the problems mentioned above, the ground field $\mathbb{R}$ can be replaced by $\mathbb{Q}_{p}$, and in fact several fields can be taken simultaneously, thus giving rise to the $S$-arithmetic setting where $S=\left\{p_{1}, \ldots, p_{s}\right\}$ is a finite set of normalized valuations on $\mathbb{Q}$, which may or may not include the infinite valuation (cf. [Sp2, Z]). The space of lattices in $\mathbb{R}^{n+1}$ is replaced there by the space of lattices in $\mathbb{Q}_{S}^{n+1}$, where $\mathbb{Q}_{S}$ is the product of the fields $\mathbb{R}$ and $\mathbb{Q}_{p_{1}}, \ldots, \mathbb{Q}_{p_{s}}$. This is the subject of the paper $[\mathrm{KT}]$, where $S$-arithmetic analogues of Theorems 1.1, 3.1 and 3.2 have been established. Similarly one can consider versions of the above theorems over local fields of positive characteristic [G2]. See also [K3] where Sprindžuk's solution [Sp2] of the complex case of Mahler's Conjecture has been generalized; the latter involves studying small values of linear forms with coefficients in $\mathbb{C}$ at real integer points.

4.7. Finally, let us mention that a generalization of Theorem 3.1 was used in $[\mathrm{BKM}]$ to estimate a measure of the set of points $\mathbf{x}$ in a ball $B \subset \mathbb{R}^{d}$ for which the system

$$
\left\{\begin{array}{l}
|\mathbf{f}(\mathbf{x}) \mathbf{q}+p|<\varepsilon \\
\left|\mathbf{f}^{\prime}(\mathbf{x}) \mathbf{q}\right|<\delta \\
\left|q_{i}\right|<Q_{i}, i=1, \ldots, n,
\end{array}\right.
$$

where $\mathbf{f}$ is a smooth map $B \rightarrow \mathbb{R}^{n}$, has a nonzero integer solution. Here $u_{\mathbf{f}(\mathbf{x})}$ has to be replaced by the matrix

$$
\left(\begin{array}{ccc}
1 & 0 & \mathbf{f}(\mathbf{x}) \\
0 & 1 & \mathbf{f}^{\prime}(\mathbf{x}) \\
0 & 0 & I_{n}
\end{array}\right),
$$

and therefore conditions (i) and (ii) of Theorem 3.1 are replaced by more complicated conditions, which nevertheless can be checked when $\mathbf{f}$ is nondegenerate. This has resulted in proving the convergence case of Khintchine-Groshev Theorem for nondegenerate manifolds, in both standard and multiplicative versions. The aforementioned estimate was also used in $[\mathrm{BBKM}]$ for the proof of the divergence case, and in [G1, G3] for establishing the convergence case of Khintchine-Groshev theorem for affine hyperplanes and their nondegenerate submanifolds.

\section{REFERENCES}

[Ba1] R. C. Baker, Metric Diophantine approximation on manifolds, J. Lond. Math. Soc. 14 (1976), 43-48. 
[Ba2] _ Dirichlet's theorem on Diophantine approximation, Math. Proc. Cambridge Phil. Soc. 83(1978), 37-59.

[BB] V. Beresnevich and V. Bernik, On a metrical theorem of W. Schmidt, Acta Arith. 75 (1996), 219-233.

[BBKM] V. Beresnevich, V. Bernik, D. Kleinbock and G. A. Margulis, Metric Diophantine approximation: the Khintchine-Groshev theorem for non-degenerate manifolds, Moscow Math. J. 2 (2002), no. 2, 203-225.

[BD] V. Bernik and M. Dodson, Metric Diophantine approximation on manifolds, Cambridge Univ. Press, Cambridge, 1999.

[BDV1] V. Beresnevich, H. Dickinson and S. Velani, Measure theoretic laws for lim sup sets, Mem. Amer. Math. Soc. 179 (2006), no. 846, 1-91.

[BDV2] - Diophantine approximation on planar curves and the distribution of rational points, Ann. Math. (to appear).

[Be] V. Beresnevich, A Groshev type theorem for convergence on manifolds, Acta Math. Hungar. 94 (2002), 99-130.

[BKM] V. Bernik, D. Kleinbock and G. A. Margulis, Khintchine-type theorems on manifolds: the convergence case for standard and multiplicative versions, Internat. Math. Res. Notices (2001), no. 9, 453-486.

[BM] B. Bekka and M. Mayer,Ergodic theory and topological dynamics of group actions on homogeneous spaces, Cambridge University Press, Cambridge, 2000.

[Bu] Y. Bugeaud, Approximation by algebraic integers and Hausdorff dimension, J. London Math. Soc. 65 (2002), no. 3, 547-559.

[D] S. G. Dani, Divergent trajectories of flows on homogeneous spaces and Diophantine approximation, J. Reine Angew. Math. 359 (1985), 55-89.

[DRV] M. Dodson, B. Rynne and J. Vickers, Dirichlet's theorem and Diophantine approximation on manifolds, J. Number Theory 36 (1990), no. 1, 85-88.

[DS1] H. Davenport and W. M. Schmidt, Dirichlet's theorem on diophantine approximation, in: Symposia Mathematica, vol. IV, INDAM, Rome, 1970, pp. 113-132.

[DS2] Dirichlet's theorem on diophantine approximation. II, Acta Arith. 16 (1969/1970), 413-424.

[G1] A. Ghosh, A Khintchine type theorem for hyperplanes, J. London Math. Soc. 72 (2005), no. 2, 293-304.

[G2] Metric Diophantine approximation over a local field of positive characteristic, J. Number Theory 124 (2007), no. 2, 454-469.

[G3] Dynamics on homogeneous spaces and Diophantine approximation on manifolds, Ph. D. Thesis, Brandeis University, 2006.

[K1] D. Kleinbock, Some applications of homogeneous dynamics to number theory, in: Smooth Ergodic Theory and Its Applications (Seattle, WA, 1999), Proc. Symp. Pure Math., vol. 68, Amer. Math. Soc., Providence, RI, 2001, pp. 639-660.

[K2] Extremal subspaces and their submanifolds, Geom. Funct. Anal. 13 (2003), no. 2, 437-466.

[K3] _ Baker-Sprindžuk conjectures for complex analytic manifolds, in: Algebraic groups and Arithmetic, Tata Inst. Fund. Res., Mumbai, 2004, pp. 539-553.

[K4] An extension of quantitative nondivergence and applications to Diophantine exponents, Trans. Amer. Math. Soc. (to appear).

$[\mathrm{KLM}]$ D. Kleinbock, E. Lindenstrauss and B. Weiss, On fractal measures and Diophantine approximation, Selecta Math. 10 (2004), no. 4, 479-523.

[KM1] D. Kleinbock and G. A. Margulis, Bounded orbits of nonquasiunipotent flows on homogeneous spaces, Amer. Math. Soc. Transl. 171 (1996), 141-172. 
[KM2] Flows on homogeneous spaces and Diophantine approximation on manifolds, Ann. Math. 148 (1998), 339-360.

[KM3] Logarithm laws for flows on homogeneous spaces, Inv. Math. 138 (1999), 451494.

[KT] D. Kleinbock and G. Tomanov, Flows on S-arithmetic homogeneous spaces and applications to metric Diophantine approximation, Comm. Math. Helv. 82 (2007), 519-581.

[KTV] S. Kristensen, R. Thorn and S. Velani, Diophantine approximation and badly approximable sets, Advances in Math. 203 (2006), 132-169.

[KW1] D. Kleinbock and B. Weiss, Badly approximable vectors on fractals, Israel J. Math. 149 (2005), 137-170.

[KW2] Friendly measures, homogeneous flows and singular vectors, in: Algebraic and Topological Dynamics, Contemp. Math. vol. 211, Amer. Math. Soc., Providence, RI, 2005, pp. 281-292.

[KW3] Dirichlet's theorem on Diophantine approximation and homogeneous flows, J. Mod. Dyn. (to appear).

[M] K. Mahler, Über das Mass der Menge aller S-Zahlen, Math. Ann. 106 (1932), 131-139.

[Ma1] G. A. Margulis, On the action of unipotent group in the space of lattices, in: Proceedings of the Summer School on group representations (Budapest 1971), Académiai Kiado, Budapest, 1975, pp. 365-370.

[Ma2] Diophantine approximation, lattices and flows on homogeneous spaces, in: A panorama of number theory or the view from Baker's garden (Zürich, 1999), Cambridge Univ. Press, Cambridge, 2002, pp. 280-310.

[MU] D. Mauldin and M. Urbański, Dimensions and measures in infinite iterated function systems, Proc. London Math. Soc. 73 (1996), no. 1, 105-154.

[PV] A. Pollington and S. Velani, Metric Diophantine approximation and 'absolutely friendly' measures, Selecta Math. 11 (2005), 297-307.

[R] M. S. Raghunathan, Discrete subgroups of Lie groups, Springer-Verlag, Berlin and New York, 1972.

[S] H. Sato, Global density theorem for a Federer measure, Tohoku Math. J. 44 (1992), no. 4, 581-595.

[Sc1] W. M. Schmidt, Metrische Sätze über simultane Approximation abhänginger Grössen, Monatsch. Math. 68 (1964), 154-166.

[Sc2] - Diophantine approximation and certain sequences of lattices, Acta Arith. 18 (1971), 195-178.

[Sp1] V. Sprindžuk, More on Mahler's conjecture, Doklady Akad. Nauk SSSR 155 (1964), 54-56 (Russian); English transl. in Soviet Math. Dokl. 5 (1964), 361-363.

[Sp2] Mahler's problem in metric number theory, Translations of Mathematical Monographs, vol. 25, Amer. Math. Soc., Providence, RI, 1969.

[Sp3] _ Achievements and problems in Diophantine approximation theory, Russian Math. Surveys 35 (1980), 1-80.

[St] A. Starkov, Dynamical systems in homogeneous spaces, FAZIS, Moscow, 1999; English translation in: Translations of Mathematical Monographs, vol. 190, Amer. Math. Soc., Providence, RI, 2000.

[SU] B. Stratmann and M. Urbański, Diophantine extremality of the Patterson measure, Math. Proc. Cambridge Phil. Soc. 140 (2006), 297-304.

[U1] M. Urbański, Diophantine approximation of self-conformal measures, J. Number Theory, 110 (2005), no. 2, 219-235.

[U2] _ Diophantine approximation for conformal measures of one-dimensional iterated function systems, Compos. Math. 141 (2005), no. 4, 869-886. 
[VV] R. C. Vaughan and S. Velani, Diophantine approximation on planar curves: the convergence theory, Inv. Math. 166 (2006), 103-124.

[W] B. Weiss, Almost no points on a Cantor set are very well approximable, Proc. R. Soc. Lond. 457 (2001), 949-952.

[Z] F. Želudevič, Simultane diophantische Approximationen abhängiger Grössen in mehreren Metriken, Acta Arith. 46 (1986), no. 3, 285-296. (German).

Dmitry Kleinbock

Dmitry Kleinbock, Department of Mathematics, Brandeis University, Waltham, MA 02454-9110

E-mail: kleinboc@brandeis.edu 